\title{
BMJ Open Risk factors for recurrent intussusception in children: a retrospective cohort study
}

\author{
Wan-liang Guo, ${ }^{1}$ Zhang-chun Hu, ${ }^{1}$ Ya-lan Tan, ${ }^{1}$ Mao Sheng, ${ }^{1}$ Jian Wang ${ }^{2}$
}

To cite: Guo W, Hu Z, Tan Y, et al. Risk factors for recurrent intussusception in children: a retrospective cohort study. BMJ Open 2017;7:e018604. doi:10.1136/ bmjopen-2017-018604

- Prepublication history for this paper is available online. To view please visit the journal online (http://dx.doi.org/10. 1136/bmjopen-2017-018604).

W-G and Z-H contributed equally.

Received 11 July 2017 Revised 28 September 2017 Accepted 18 0ctober 2017

\section{(a) CrossMark}

${ }^{1}$ Department of Radiology, Children's Hospital of Soochow University, Suzhou, China ${ }^{2}$ Department of Pediatric Surgery, Children's Hospital of Soochow University, Suzhou, China

Correspondence to

Dr Jian Wang;

wangjiansuzhou@163.com

\begin{abstract}
Objective The aim of this study was to assess the frequency of clinical features and pathological lead points in recurrent intussusception, with a special focus on the risk factors that lead to recurrent intussusception. Design This is a retrospective cohort study. A 5-year retrospective study was performed between January 2012 and July 2016 in the Children's Hospital of Soochow University, Suzhou, China, to determine the clinical features and pathological lead points of recurrent intussusception.
\end{abstract}

Setting This is a retrospective chart review of recurrent intussusception cases in a large university teaching hospital.

Participants The medical records were obtained for 1007 cases with intussusception, including demographics, clinical signs and symptoms, imaging and recurrence times if available.

Interventions Univariate and multivariate logistic regression analyses were used to measure significant factors affecting recurrent intussusception and recurrent intussusception with pathological lead points.

Results There were 481 total episodes of recurrence in 191 patients. Among these, 87 had one recurrence and 104 had multiple recurrences. After comparing recurrent and non-recurrent intussusception cases using univariate analysis, it was determined that the factors associated with recurrent intussusception were age ( $>1$ year), duration of symptoms ( $\leq 12$ hours), the lack of bloody stool, paroxysmal crying or vomiting, the mass location (right abdomen) and pathological lead point $(P<0.05)$. Age $(>1$ year), duration of symptoms ( $\leq 12$ hours), the absence of vomiting, mass location (right abdomen) and pathological lead point were significantly independently predictive of recurrent intussusception. The factors associated with recurrent intussusception with lead points present were vomiting and mass location in the right abdomen $(P<0.05)$. Vomiting and mass location (left abdomen) were significantly predictive of recurrent intussusception with lead points.

Conclusions Age ( $>1$ year), symptom duration ( $\leq 12$ hours), the absence of vomiting, mass location (right abdomen) and pathological lead points were significantly predictive of recurrent intussusception. Vomiting and mass location (left abdomen) were significantly predictive of recurrent intussusception with lead points.

\section{Strengths and limitations of this study}

- A retrospective cohort study was carried out in our hospital over a 5 -year period. A relative large number of 191 recurrent intussusceptions was analysed. A stepwise logistic regression analysis of 1007 cases was performed to assess the independent predictors of recurrent intussusception.

- Age (>1 year), symptom duration ( $\leq 12$ hours), the absence of vomiting, mass location (right abdomen) and pathological lead points were significantly predictive of recurrent intussusception.

- This study has several limitations. First, the current study is subject to its retrospective design and potential selection bias. Also, the conclusions are based on observations from a single children's centre so a prospective, multicentre study is needed to further evaluate factors that predict recurrent intussusception.

\section{INTRODUCTION}

Intussusception is one of the most frequent causes of intestinal obstruction in infants and children, and most intussusception cases are idiopathic. Previous studies have reported that pathological lead points, such as Meckel's diverticulum, duplication, polyp and tumours, can lead to recurrent intussusception in children. ${ }^{1-5}$ Once intussusception has been detected, an air enema or hydrostatic reduction can be used to reduce the intussusception, with an excellent outcome. ${ }^{6}$

The recurrence of intussusception is relatively common regardless of the method of reduction. ${ }^{7}$ Some authors have reported that approximately $10 \%$ of patients (range: $8 \%-15 \%$ ) suffer from recurrent intussusception. ${ }^{8}$ Clinically, the early diagnosis and management of recurrent intussusception is challenging to paediatricians, radiologists and paediatric surgeons. Although a previous study reported that lead points can precipitate recurrent intussusception, reliable risk factors for most cases of recurrence have not been identified. ${ }^{10}$ 


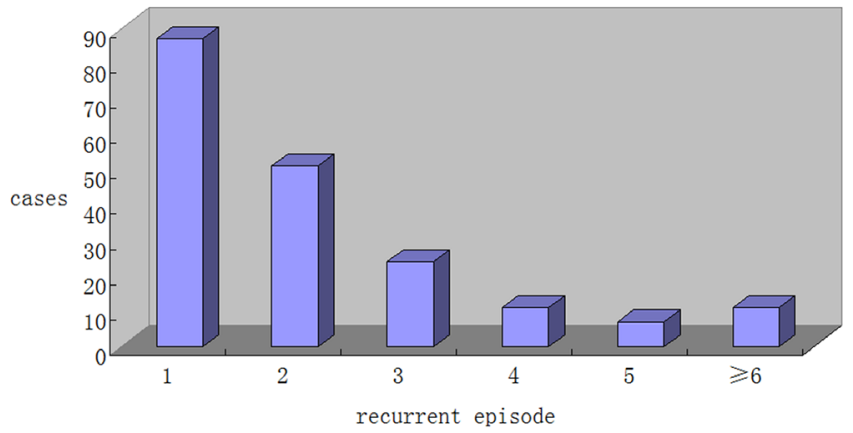

Figure 1 Distribution of recurrent episode times of intussusceptions (45.5\% had one episode of recurrence).

To date, very few studies have focused on the risk factors for recurrent intussusception. Therefore, a 5-year retrospective study was performed between January 2012 and July 2016 in the Children's Hospital of Soochow University, Suzhou, China, to determine the clinical features and pathological lead points of recurrent intussusception, with a special focus on the risk factors for intussusception recurrence.

\section{METHODS}

\section{Study design}

This retrospective study included the medical records and radiographs of 1007 paediatric cases with intussusception who presented to the Children's Hospital of Soochow University between January 2012 and July 2016. The final diagnosis was made by ultrasound or air enema. Once the diagnosis of intussusception was made, informed consent was obtained from the parents or designated guardians of each child, and air enema reduction was used to manage the intussusception. The air enema reduction procedure was performed under fluoroscopy by experienced paediatric radiologists in the Children's Hospital of Soochow University. None of the patients were sedated before the air enema, which had incipient pressure of $6 \mathrm{Kpa}$, and the upper limit was $12 \mathrm{Kpa}$. If the air enema reduction failed three times, the patients then underwent surgery. Demographic data, medical history, clinical signs and symptoms, and images were collected for each case when available. Recurrent cases were identified by ultrasound or air enema confirmation and combined by medical history of air enema or surgical reduction.

\section{Study groups}

Recurrent intussusception was defined as the recurrence of intussusception after air enema reduction or surgery. Non-recurrent intussusception was defined as the cases that were successfully reduced after air enema or surgery without recurrence. Cases of non-recurrent intussusception were used as the control group when analysing the risk factors for recurrent intussusception. A total of 1007 cases with intussusception (113 cases requiring surgery) were separated into two groups according to age (0-12 months and $>1$ year), gender, symptom duration, bloody stool, paroxysmal crying, vomiting, the location of the mass and the pathological lead points were analysed. All of the information was obtained at the time of the first reduction by air enema or surgery. Every case was followed up for 1 year from the time of the first episode.

\section{Patterns of recurrence}

Single-recurrent intussusception was defined as the cases that recurred only once after air enema reduction or surgery. Multiple-recurrent intussusception was defined as the cases that recurred at least twice after air enema reduction or surgery.

\section{Recurrent intussusception with lead points present}

Recurrent intussusception with lead points present was defined as the cases after air enema reduction with confirmed lead points by surgery. Recurrent intussusception with lead points unknown was defined as the cases after air enema reduction or surgery in which lead points could not be found.

\section{Mass location}

The mass location in the right abdomen was defined as the cases in which the mass was on the right side of the spine. The left abdomen was defined as the cases in which the mass was on the left side of the spine.

\section{STATISTICAL ANALYSIS}

Data are presented as numbers (n) and percentages. Univariate comparisons were made using non-parametric one-way Wilcoxon's rank sum, $\chi^{2}$ and Fisher's exact tests depending on the statistical distribution. The factors that contribute to recurrent intussusception using air enema or surgery were analysed using logistic regression analysis with Statistical Analysis Software (SAS) V.8.1. P values $<0.05$ were considered to be statistically significant.

\section{RESULTS}

\section{Clinical characteristics of recurrent intussusception cases}

There were 481 total episodes of recurrence in 191 patients. The median age of the 191 patients was 2 years (range: 80 days-11 years). A total of 117 of the 191 patients were male $(61.26 \%)$. Among the recurrent cases, 87 had one episode of recurrence, 51 had two recurrences, 24 had three recurrences, 11 had four recurrences, 7 had 5 recurrences and 11 had more than six recurrences (figure 1). Sixty-five cases of recurrence occurred within 24 hours, 28 occurred between 24 hours and 1 week, 14 occurred between 1 week and 1 month, 63 occurred between 1 month and 6 months and 21 occurred between 6 months and 1 year (figure 2 ). The baseline characteristics and procedural details of the patients are shown in table 1 .

\section{Predictors of recurrent intussusception}

Table 2 shows the clinical characteristics of the recurrent intussusception cases. When recurrent and non-recurrent 


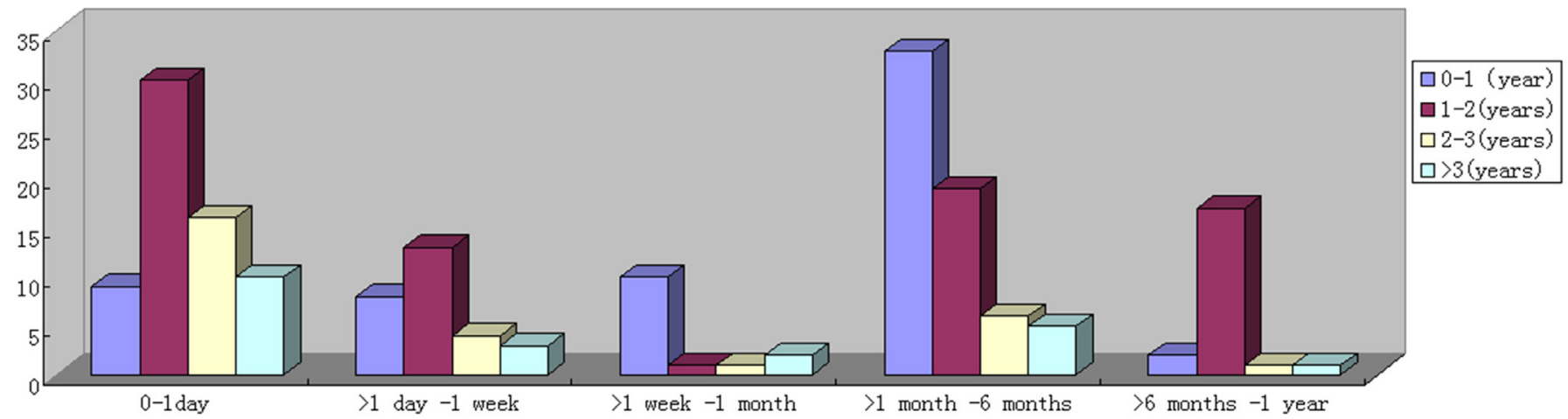

Figure 2 Distribution of recurrent episode time points among four age groups (74.3\% recurrent cases are below 2 years old).

intussusception cases were compared using univariate analysis, the factors associated with recurrent intussusception were age ( $>1$ year), symptom duration ( $\leq 12$ hours), mass location (right abdomen), pathological lead point, the absence of bloody stool or vomiting $(\mathrm{P}<0.05)$. There were no significant differences in gender and paroxysmal crying between the two groups $(\mathrm{P}>0.05)$. Logistic regression analysis was performed to assess the independent predictors of recurrent intussusception among the 1007 intussusception cases. Age ( $>1$ year), symptom duration ( $\leq 12$ hours), the absence of vomiting, mass location (right abdomen) and pathological lead points were significantly predictive of recurrent intussusception (table 3). The logistic regression model was consistent with findings reported by Hosmer and Lemeshow using the goodness of fit test $(\mathrm{P}=0.8643)$.

\section{Predictors of recurrent intussusception with lead points}

Table 4 shows the clinical characteristics of recurrent intussusception cases with lead points. Among the recurrent intussusception cases with lead points, four were cases with Meckel's diverticulum, eight were cases with duplication, four had polyps and three had tumours. When recurrent cases with and without lead points were compared using univariate analysis, the factors associated with recurrent intussusception with lead points were vomiting, bloody stool and mass location (left abdomen) $(\mathrm{P}<0.05)$. There were no significant differences in age, symptom duration and paroxysmal crying between the two groups $(\mathrm{P}>0.05)$. Logistic regression analysis was then performed to assess the independent predictors of recurrent intussusception with lead points among 191 intussusception cases. Vomiting and mass location (left abdomen) were significantly predictive of recurrent intussusception with lead points (table 5). The logistic regression model was consistent with findings reported by Hosmer and Lemeshow using the goodness-of-fit test $(p=0.6422)$.

\section{Patterns of recurrence}

There was no seasonal pattern of recurrence, and the early recurrence rate (recurrence within 24hours) was $6.2 \%$. Table 6 shows the clinical characteristics and the numbers of recurrence in the recurrent intussusception cases. Using univariate analysis to compare single and multiple recurrence intussusception cases, there were no significant differences in age ( $>1$ year), symptom duration ( $\leq 12$ hours), mass location (right abdomen), pathological lead points and the absence of bloody stool, paroxysmal crying and vomiting $(\mathrm{P}>0.05)$.

\section{DISCUSSION}

\section{General findings in recurrent intussusception}

Recurrent intussusception is relatively common. Delays in the diagnosis of intussusception and reduction can lead to serious complications, including bowel ischaemia, perforation and peritonitis. However, the early diagnosis and management of recurrent intussusception is challenging to paediatricians, radiologists and paediatric surgeons. Previous studies have noted that the rate of recurrence

Table 1 Age and sex distribution of recurrence cases from January 2012 to July 2016

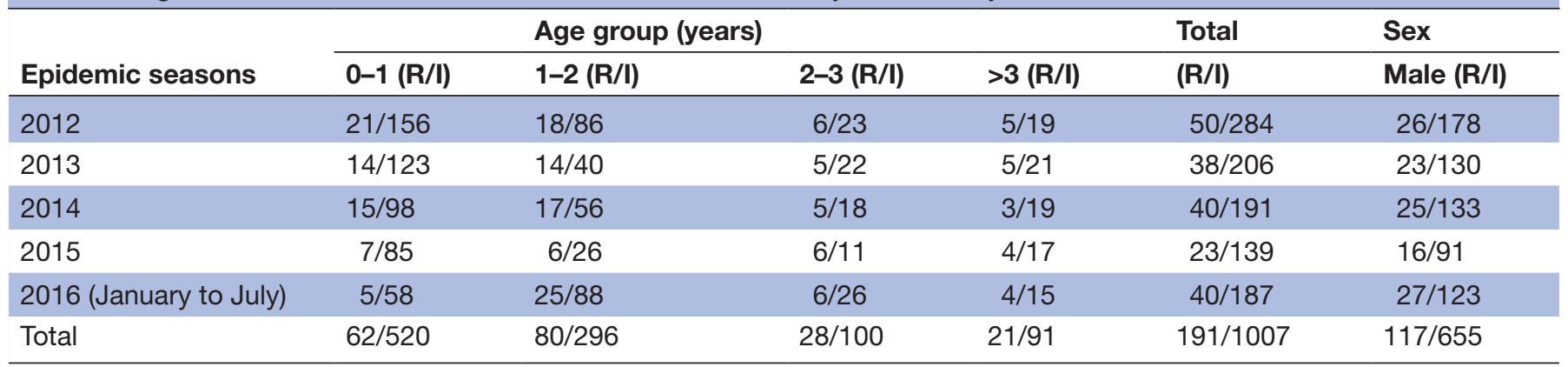

I, all intussusception cases; R, recurrent intussusception. 
Table 2 Comparison of recurrent with non-recurrent intussusceptions

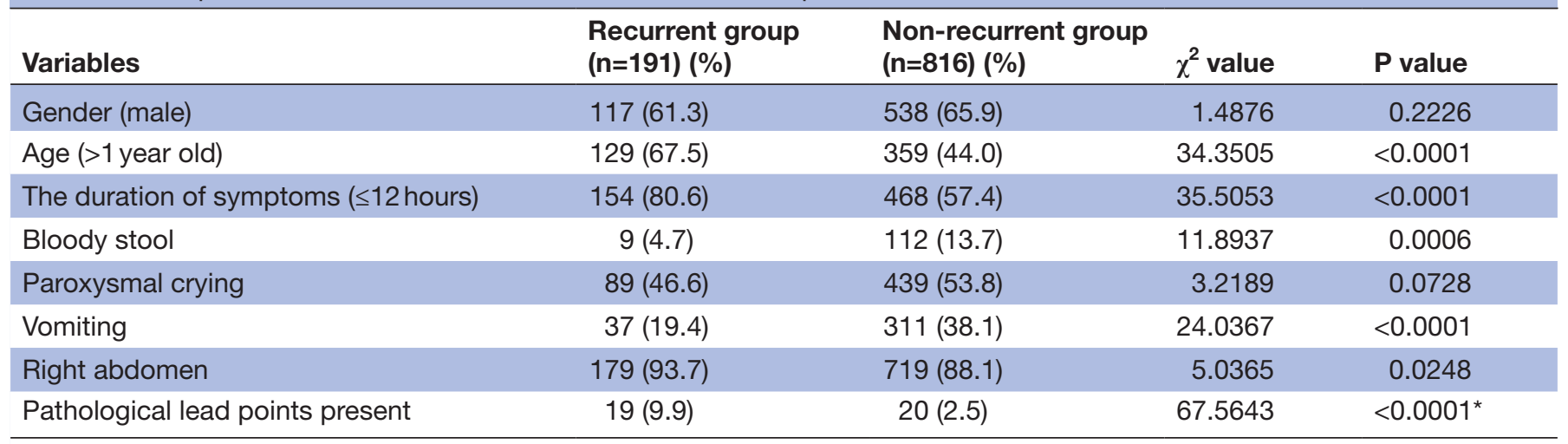

${ }^{\star}$ Fisher's exact test.

of intussusception ranges from $8 \%$ to $15 \%,{ }^{8}$ which is a little lower than the current results. A possible reason for this difference is the high rate of surgical reduction in their studies. ${ }^{7}$ Daneman et al reported 69 recurrent intussusception cases over 17 years. They compared patients with recurrence that underwent air enema and barium enema, and they found that the rates and patterns of recurrence did not change with the different management strategies. Furthermore, they emphasised that the presence of pathological lead points should always be considered in children with recurrent intussusception. ${ }^{10}$ Champoux et al reported 23 recurrent intussusception cases and attempted to determine the risk factors associated with recurrent intussusception. They found that although recurrent intussusception cannot be predicted by presenting features or symptoms, operative reduction after failed reduction using a barium enema reduced the risk of recurrent intussusception. ${ }^{11}$ However, the sample size in the above studies was smaller than in the present study. Therefore, a 5-year retrospective study was undertaken to determine the risk factors that influence intussusception recurrence in children.

\section{Factors for detecting recurrent intussusception}

To investigate the factors that might influence recurrent intussusception, the clinical characteristics, mass location and pathological findings of children with intussusception

Table 3 The stepwise regression model of risk factors of recurrent intussusception

\begin{tabular}{lllr}
\hline Variables & OR & $\mathbf{9 5 \%}$ Cl & P value \\
\hline Age & 2.117 & 1.476 to 3.035 & $<0.0001$ \\
$\begin{array}{l}\text { The duration of } \\
\text { symptoms }\end{array}$ & 2.868 & 1.929 to 4.262 & $<0.0001$ \\
Vomiting & 0.500 & 0.324 to 0.770 & 0.0016 \\
\hline $\begin{array}{l}\text { Right abdomen } \\
\text { Pathological lead } \\
\text { point present }\end{array}$ & 2.076 & 1.047 to 4.113 & 0.0364 \\
\hline
\end{tabular}

Hosmer and Lemeshow goodness-of-fit test: $\chi^{2}$ value $=2.5382, \mathrm{P}=0.8643$. were described. Univariate analysis indicated that patient age, symptom duration, bloody stool, vomiting, mass location and pathological lead points may affect the recurrence of intussusception in children. Multiple logistic regression analysis confirmed that age, symptom duration, vomiting, mass location, and pathological lead points were independent factors for predicting the recurrence of intussusception in children.

Patients with intussusception who are older than 1 year of age may be more likely to experience the recurrence of intussusception. In the present study, $67.5 \%$ of the recurrent cases were aged $>1$ year. Wang et al reported 127 cases of recurrent intussusception, and they found that the rate of recurrence was lower in patients aged $<1$ year than in those aged $>1$ year. ${ }^{12}$ This is similar to the current results. Esmaeili-Dooki et al reported 38 recurrent cases, and they found that nearly $50 \%$ of the children with recurrent intussusception were younger than 1 year at the first episode, and recurrent intussusception decreased to $7.9 \%$ in older children. ${ }^{7}$ These results differ from the current results. A possible reason for this is that the rate of surgical reduction in children aged $<1$ year was higher in the study by Esmaeili-Dooki et al than in the current study, and the risk of recurrence is lower in children receiving operative reduction compared with non-operative reduction. ${ }^{8}$ Another reason is that the current study used multiple factor analysis and had a relatively large number of recurrent cases. We believe that the current study will have a significant impact on efforts to find novel strategies for patients aged $>1$ year with intussusception.

Some clinical characteristics are associated with recurrence. In the present study, symptom duration ( $\leq 12$ hours), the absence of vomiting and mass location (right abdomen) were independent predictors of recurrent intussusception. Justice et al reported 85 cases of recurrent intussusception and found that the incidence was higher in patients with a mass location in the right side of the colon compared with the left side of the colon. ${ }^{13}$ Champoux et al reported 23 recurrent intussusception cases, and they concluded that patients who experience recurrent intussusception have fewer symptoms with a shorter duration. ${ }^{11}$ The current study also found 
Table 4 Comparison of recurrent intussusception with or without pathological lead points present in recurrent cases

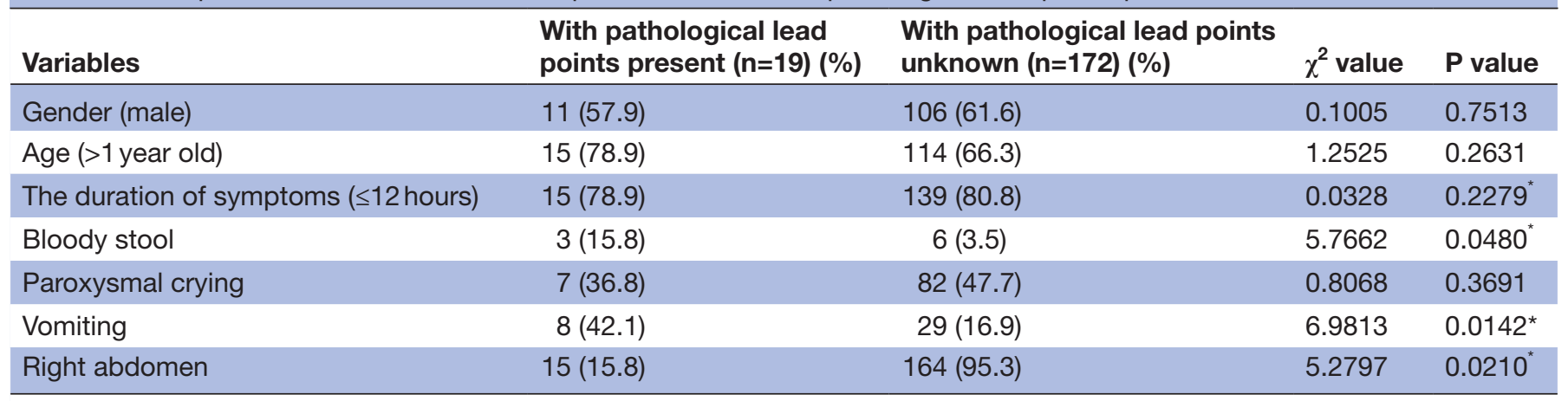

${ }^{*}$ Fisher's exact test.

that recurrent cases have a lower incidence of vomiting and a shorter duration than non-recurrent cases, which is similar to the above results.

\section{Factors for detecting recurrent intussusception with pathological lead points}

Intussusception cases caused by pathological lead points might more easily lead to recurrence. In this series, pathological lead points were detected in $9.9 \%$ of the patients, in $3 \%$ of the children who had one recurrence and $6 \%$ of the children who had multiple recurrences. Multiple logistic regression confirmed that the location and pathological lead points are independent factors for the recurrence of intussusception in children. Daneman et al reported that pathological lead points were present in $8 \%$ of all of the children with recurrent intussusception, in $4 \%$ of the children with one episode of recurrence and in $14 \%$ of the children with multiple recurrences. ${ }^{10}$ Niramis et al reported 1340 intussusception cases and 75 recurrent intussusception cases, and they found that pathological lead points were noted in $7(9.3 \%)$ of the 75 patients with recurrent intussusception compared with $48(3.8 \%)$ of the 1265 patients with intussusception that did not recur. This represented an approximate threefold increase in the incidence of lead points in children with recurrent intussusception. ${ }^{8}$ The above study is similar to the current results. However, Fisher et al reported that the rate of pathological lead points was similar among patients with one, two or three or more episodes of intussusception. ${ }^{14}$ The epidemiology of lead points may depend on the exposure to infectious diseases, and thus may differ substantially between Asia and North America. ${ }^{15}$ Therefore, based on the present

Table 5 The stepwise regression model of risk factors of recurrent intussusception with pathological lead points present

\begin{tabular}{llll}
\hline Variables & OR & $\mathbf{9 5 \%} \mathbf{C l}$ & P value \\
\hline Vomiting & 3.004 & 1.056 to 8.548 & 0.0393 \\
Right colon & 0.165 & 0.043 to 0.638 & 0.0090 \\
\hline
\end{tabular}

Hosmer and Lemeshow goodness-of-fit test: $\chi^{2}$ value $=0.2591$, $\mathrm{P}=0.6108$. study, which combined univariate and multiple logistic regression analysis, it appears that the recurrence of intussusception is a risk factor for harbouring pathological lead points. In addition, the current study found that recurrent intussusception cases with pathological lead points may more often experience vomiting and a mass in the left abdomen. Multiple logistic regression analysis also confirmed that vomiting and mass location were independent factors for predicting the recurrence of intussusception with pathological lead points in children. This has not been reported previously and has not yet been confirmed.

\section{Patterns of recurrence}

A total of $45.5 \%$ of the recurrent cases experienced only one recurrence, and $54.5 \%$ experienced multiple recurrences. Univariate analysis showed there were no significant differences in age, symptom duration, bloody stool, paroxysmal crying, vomiting, mass location and pathological lead points between the single and multiple recurrence intussusception groups. In addition, there was no seasonal pattern of recurrence, and the early recurrence rate (recurrence within 24 hours) was $6.5 \%$. Gilmore et al reviewed 117 paediatric patients with intussusception during a 15-year period, and they found that the early recurrence rate was $5.3 \%,{ }^{16}$ while McDermott et al reported that the early recurrence rate was $7 \% .{ }^{17}$ The above studies are consistent with the present study, suggesting that intussusception cases should be carefully observed during the early period after air enema reduction.

\section{LIMITATIONS}

The greatest limitations of the current study are its retrospective design and potential selection bias, as some of the cases with pathological lead points were not detected during the observation period. Also, the conclusions are based on observations from a single children's centre so a prospective, multicentre study is needed to further evaluate factors that predict recurrent intussusception. 
Table 6 Comparison of different recurrence numbers of intussusception

\begin{tabular}{|c|c|c|c|c|}
\hline Variables & $\begin{array}{l}\text { Single recurrence group } \\
(\mathrm{n}=87)\end{array}$ & $\begin{array}{l}\text { Multiple recurrence group } \\
(n=104)\end{array}$ & $\chi^{2}$ value & $P$ value \\
\hline Gender (male) & 55 & 62 & 0.2591 & 0.6107 \\
\hline The duration of symptoms ( $\leq 12$ hours) & 67 & 87 & 1.3382 & 0.2474 \\
\hline Bloody stool & 6 & 3 & 0.9222 & $0.3369^{*}$ \\
\hline Right abdomen & 84 & 95 & 2.1802 & 0.1398 \\
\hline Pathological lead points present & 5 & 14 & 3.1471 & 0.0761 \\
\hline
\end{tabular}

${ }^{\star}$ Fisher's exact test.

\section{CONCLUSIONS}

In conclusion, a logistic regression analysis was performed to assess the independent predictors of recurrent intussusception among 1007 intussusception cases. Age ( $>1$ year), symptom duration ( $\leq 12$ hours), the absence of vomiting, mass location (right abdomen) and pathological lead points were significant predictors of recurrent intussusception. Vomiting and mass location (left abdomen) were significantly predictive of recurrent intussusception with lead points. No significant clinical differences were found between the single and multiple recurrence intussusception cases.

Contributors WG and JW designed the study; WG, ZH, YT and MS collected data; WG and JW analysed the data; WG and ZH wrote the manuscript; and all authors read and approved the final version of manuscript.

Funding This work was supported by Jiangsu Province Health and Family Planning Projects (H201519), Fundamental and Application Research in Health Care of Suzhou (SYS201762) and Jiangsu Province Social Development Program Standardized Diagnosis and Treatment of Key Disease in Clinic (BE2015643).

Competing interests None declared.

Ethics approval This study was approved by the Institutional Review Board of the Children's Hospital of Soochow University.

Provenance and peer review Not commissioned; externally peer reviewed.

Data sharing statement No additional data are available.

Open Access This is an Open Access article distributed in accordance with the Creative Commons Attribution Non Commercial (CC BY-NC 4.0) license, which permits others to distribute, remix, adapt, build upon this work non-commercially, and license their derivative works on different terms, provided the original work is properly cited and the use is non-commercial. See: http://creativecommons.org/licenses/by-nc/4.0/

(C) Article author(s) (or their employer(s) unless otherwise stated in the text of the article) 2017. All rights reserved. No commercial use is permitted unless otherwise expressly granted.

\section{REFERENCES}

1. Saka R, Sasaki T, Matsuda I, et al. Chronic ileocolic intussusception due to transmural infiltration of diffuse large B cell lymphoma in a 14-year-old boy: a case report. Springerplus 2015;4:366.

2. Dias AR, Lopes RI, do Couto RC, et al. Ileal duplication causing recurrent intussusception. J Surg Educ 2007;64:51-3.

3. Kim KH, Kang KA, Lim JH, et al. Inverted Meckel diverticulum as a lead point of small bowel intussusception: misinterpreting case as a lipoma. Clin Imaging 2016;40:840-2.

4. Basara I, Canda AE, Sagol O, et al. Intussusception and perforation due to an inflammatory fibroid polyp located in the ileum. Wien Klin Wochenschr 2016;128:731-4.

5. Siminas S, Qasem E, Shukla R, et al. Inflammatory fibroid polyp: a rare benign tumor of the alimentary tract in children presenting as intussusception-case report and review of literature. European J Pediatr Surg Rep 2014;2:16-19.

6. Beres AL, Baird R. An institutional analysis and systematic review with meta-analysis of pneumatic versus hydrostatic reduction for pediatric intussusception. Surgery 2013;154:328-34.

7. Esmaeili-Dooki MR, Moslemi L, Hadipoor A, et al. Pediatric intussusception in northern iran: Comparison of recurrent with nonrecurrent cases. Iran J Pediatr 2016;26:e3898.

8. Niramis R, Watanatittan S, Kruatrachue A, et al. Management of recurrent intussusception: nonoperative or operative reduction? $J$ Pediatr Surg 2010;45:2175-80.

9. Fecteau A, Flageole H, Nguyen LT, et al. Recurrent intussusception: safe use of hydrostatic enema. J Pediatr Surg 1996;31:859-61.

10. Daneman A, Alton DJ, Lobo E, et al. Patterns of recurrence of intussusception in children: a 17-year review. Pediatr Radiol 1998;28:913-9.

11. Champoux AN, Del Beccaro MA, Nazar-Stewart V. Recurrent intussusception. Risks and features. Arch Pediatr Adolesc Med 1994;148:474-8.

12. Wang $\mathrm{Z}, \mathrm{He} \mathrm{QM}$, Zhang $\mathrm{H}$, et al. Intussusception patients older than 1 year tend to have early recurrence after pneumatic enema reduction. Pediatr Surg Int 2015;31:855-8.

13. Justice FA, Nguyen LT, Tran SN, et al. Recurrent intussusception in infants. J Paediatr Child Health 2011;47:802-5.

14. Fisher JG, Sparks EA, Turner CG, et al. Operative indications in recurrent ileocolic intussusception. J Pediatr Surg 2015;50:126-30.

15. Bines JE, Ivanoff $B$, Justice $F$, et al. Clinical case definition for the diagnosis of acute intussusception. J Pediatr Gastroenterol Nutr 2004;39:511-8.

16. Gilmore AW, Reed M, Tenenbein M. Management of childhood intussusception after reduction by enema. Am J Emerg Med 2011;29:1136-40.

17. McDermott VG, Taylor T, Mackenzie S, et al. Pneumatic reduction of intussusception: clinical experience and factors affecting outcome. Clin Radiol 1994;49:30-4. 